\title{
种植制度对江苏省棉田杂草群落影响的研究
}

\author{
强 胜 $^{1}$ 沈俊明 ${ }^{2}$ 张成群 $^{1} \quad$ 邵耕耘 $^{3}$ 胡金良 $^{1}$ 王风良 ${ }^{4}$ \\ （1 南京农业大学杂草研究室 南京 210095）（2 江苏省南通市沿江地区农科所 南通 226541） \\ （3 江苏省宝应市植保站 宝应 225800）（4 江苏省大丰市植保站 大丰 224000）
}

\begin{abstract}
摘 要 经连续 5 年对江苏省主棉区水旱轮作及旱连作两种种植制度下的棉田杂草种群密度及草害优势度级数 进行定田调查, 通过时间和空间上的比较统计分析, 研究了棉田杂草群落演替趋势及草害发生规律。研究结果表 明 种植制度与江苏省棉田杂草群落的组成和草害发生程度紧密相关。在水旱轮作棉田中, 随着棉花(Gossypium hirsutum) 种植年数的增加, 喜湿性杂草稗草 (Echinochloa crusgalli) 等优势度值逐渐减少, 喜旱性杂草马唐 (Digitaria sanguinalis) 等优势度值逐渐提高, 它们之间存在显著的线性关系。旱连作棉田以喜旱性杂草为主, 发生量较大 草 害较重, 年际间杂草群落变化较小。另外, 水旱轮作和旱连作棉田之间的杂草群落结构和种群数量差异显著。而 地理区域差异的影响明显小于轮作制度差异导致的影响。
\end{abstract}

关键词＼cjkstart棉田杂草群落 演替 种植制度 江苏省

\section{THE INFLUENCE OF CROPPING SYSTEMS ON WEED COMMUNITIES IN THE COTTON FIELDS OF JIANGSU PROVINCE}

\author{
QIANG Sheng $^{1} \quad$ SHEN Jun-Ming ${ }^{2}$ ZHANG Cheng-Qun ${ }^{1}$ SHAO Geng-Yun ${ }^{3}$ \\ HU Jin-Liang ${ }^{1}$ and WANG Feng-Liang ${ }^{4}$ \\ (1 Weed Research Laboratory, Nanjing Agricultural University, Nanjing 210095 , China) \\ (2 Nantong Agricultural Institute, Nantong, Jiangsu Province, Nantong 226541, China) \\ (3 Baoying Plant Protective Station, Jiangsu Province, Baoying 225800 , China) \\ (4 Dafeng Plant Protective Station, Jiangsu Province , Dafeng 224000 , China)
}

\begin{abstract}
The overall weed infestation indexes and density of all weed populations occurring in both the ricecotton crop rotation and continuous dry-cropping systems of the main cotton belts in Jiangsu Province were surveyed over five years continuously. The succession patterns of weed communities and weed occurrence were studied through statistical analysis in space and time. The results showed that there was a close relationship between the structure of weed community and the level of weed infestation and cropping systems. Under the rice-cotton crop rotation system, the density of hydrophilous weeds such as Echinochloa crusgalli , Alternanthera philoxeroides and Malachium aquaticum was the highest, while that of xerophilous weeds (e.g. , Digitaria sanguinalis) was lowest. However, there was marked linear relationship between abundance and time , with abundance of hydrophilous weeds (e.g. ,E. crusgalli) decreasing, and that of xerophilous weeds as (e. g. , D. sanguinalis ) increasing with time, when cotton was grown continuously in the fields with the rice-cotton rotation system. After four years continuous cotton growing, the structure of weed community seemed to tend to stable condition. In the cotton fields with cotton cropping system, there were dense density of xerophilous weeds as dominants and no marked change of weed community structure between different years. Moreover , the marked difference of weed community structure and weed density occurred between two different cropping systems. The influence of cropping systems on weed community was much more significant than that of geographical factors.
\end{abstract}

Key words Weed community in the cotton field, Succession, Cropping system , Jiangsu Province

在江苏省主要棉花 (Gossypium hirsutum) 种植区 内大致存在水旱和旱旱两种主要的种植制度, 且在 每个棉花种植区其种植制度有相当部分是相互交错 的(江苏农业地理编写组，1979)。此前, 通过对江
苏全省主棉区的棉田杂草进行了定量的调查研究， 表明轮作制度是制约棉田杂草群落和草害的主要因 素之一(强胜等,1997；1999；2000)。上述研究，仅 从全省的空间角度, 揭示了棉田杂草群落空间分布 
规律及其影响因素。但是, 针对轮作制度对棉田杂 草群落影响的专门研究报道尚少, 特别是从时间序 列上开展的研究还未见报道 (朱文达等, 1993)。国 外的相关研究多集中在玉米 (Zea mays)、大豆 (Glyncine max) 等旱地作物, 水旱轮作尚少见报道 (Zanin ,1997)。本研究对江苏省沿江棉区的南通、里 下河棉区的宝应、沿海棉区的大丰等三地的旱连作 棉田与水旱轮作棉田的杂草群落进行连续 5 年定田 调查研究, 试图从时间序列和空间序列上揭示种植 制度与江苏省棉田杂草群落和草害发生规律之间的 关系, 为江苏农业种植制度的制定和棉田杂草防除 提供理论依据。

\section{1 调查研究范围及方法}

从 1996 ～ 2000 年, 连续 5 年在江苏省沿海、沿 江、里下河棉区的大丰市大中镇和白驹镇、南通市农 科所、宝应县曹甸镇和夏集镇等地分别取水旱轮作 棉田及旱连作棉田各 3 块, 调查田间杂草。大丰点 1997 和 2000 年移栽前曾用氟乐灵化学除草, 结合常 规人工除草 $1 \sim 2$ 次; 宝应点 1999 和 2000 年地膜覆 盖 结合一次雍根除草; 其余年份与南通点相同, 常 规人工除草 $2 \sim 3$ 次; 每块田中取 5 个样本点, 每个 样本点大小为 $1 \mathrm{~m}^{2}$ 。

在棉花移栽后 $20 \mathrm{~d}$ (现蕾初期)、蕾铃期和裂铃 吐絮期分别调查记载每个样本的杂草种类及其株 数。还根据杂草的相对高度、多度和相对盖度，采用 七级目测法分级标准算出每种杂草的优势度值 (强 胜等 ,1990)。

以平均每个样本点出现超过一株的杂草作为分
析指标。应用 Microsoft Excel 对水旱轮作田连续 5 年种植棉花过程中各种杂草优势度值与水改旱种植 年数之间的关系进行直线回归分析, 并利用 SPSS 11.0 统计软件对水旱轮作田 5 年间的田间杂草优 势度值变化情况进行差异显著性分析, 利用 STST 统 计软件对南通、宝应、大丰三地水旱轮作与旱连作两 种种植制度下的杂草发生数量进行两项分组资料方 差分析。

\section{2 结果与分析}

在旱连作田及水旱轮作种植过程中，棉田中主 要杂草连续 5 年的优势度值及其优势度值在各年份 间的差异显著性分析结果见表 1 。从表 1 中可以看 出, 水旱轮作种植制度和旱连作种植制度下的棉田 杂草群落结构明显不同。该地区水旱轮作种植制度 下棉田中的主要杂草有稗草 (Echinochloa crusgalli)、 马唐 (Digitaria sanguinalis)、千金子 (Leptochloa chinensis)、水花生 (Alternanthera philoxeroides)、牛繁缕 (Malachium aquaticum) 和鳢肠 (Eclipta prostrata)。其 中以喜湿性杂草为主, 兼有喜旱性杂草马唐。而该 地区旱连作种植制度下棉田中的主要杂草是马唐、 刺儿菜 (Cephalanoplos segetum)、虮子草 (Leptochloa panicea) 和鳢肠, 以喜旱性杂草为主。从空间序列 看 江苏省主要棉区水旱轮作种植制度下的棉田杂 草群落结构趋于一致, 以稗、马唐、鳢肠和千金子为 优势杂草; 而旱连作种植制度下的棉田杂草群落结 构随不同的地理区域而有所不同, 但以喜旱性杂草 为主 (强胜等, $1997 ; 1999 ; 2000)$ 。

表 1 不同年份棉田主要杂草优势度

Table 1 Dominances of main weeds in cotton fields over five years

\begin{tabular}{|c|c|c|c|c|c|c|}
\hline \multirow{2}{*}{$\begin{array}{c}\text { 种植制度 } \\
\text { Cropping system }\end{array}$} & \multirow{2}{*}{$\begin{array}{l}\text { 杂草种名 } \\
\text { Weed species }\end{array}$} & \multicolumn{5}{|c|}{ 综合优势度 Overall dominance (OD) } \\
\hline & & 1996 & 1997 & 1998 & 1999 & 2000 \\
\hline \multirow{6}{*}{$\begin{array}{l}\text { 水旱轮作 Rotation of } \\
\text { cotton and rice }\end{array}$} & 稗草 Echinochloa crusgalli & $3.4^{\mathrm{a}}$ & $1.3^{\mathrm{b}}$ & $0.92^{\mathrm{b}}$ & $0.7^{\mathrm{b}}$ & $0.52^{\mathrm{b}}$ \\
\hline & 马唐 Digitaria sanguinalis & $0.24^{\mathrm{d}}$ & $1^{\mathrm{cd}}$ & $2^{\mathrm{bc}}$ & $3.8^{\mathrm{a}}$ & $2.4^{\mathrm{b}}$ \\
\hline & 千金子 Leptochloa chinensis & $0.62^{\mathrm{cd}}$ & $1.52^{\mathrm{bc}}$ & $1.82^{\mathrm{b}}$ & $3.4^{\mathrm{a}}$ & $0.02^{\mathrm{d}}$ \\
\hline & 水花生 Alternanthera philoxeroides & $1.4^{\mathrm{a}}$ & $1.6^{\mathrm{a}}$ & $0.32^{\mathrm{b}}$ & $0.02^{\mathrm{b}}$ & $0.42^{\mathrm{b}}$ \\
\hline & 牛繁缕 Malachium aquaticum & $3.6^{\mathrm{a}}$ & $0.04^{\mathrm{b}}$ & $0.04^{\mathrm{b}}$ & $0^{\mathrm{b}}$ & $0^{\mathrm{b}}$ \\
\hline & 鳢肠 Eclipta prostrata & $0.08^{\mathrm{a}}$ & $0.32^{\mathrm{a}}$ & $0.52^{\mathrm{a}}$ & $0.14^{\mathrm{a}}$ & $0.02^{\mathrm{a}}$ \\
\hline \multirow{4}{*}{$\begin{array}{l}\text { 旱连作 Continuous dry } \\
\text { cropping system }\end{array}$} & 马唐 Digitaria sanguinalis & $2.4^{\mathrm{a}}$ & $2.6^{\mathrm{a}}$ & $2.4^{\mathrm{a}}$ & $3.4^{\mathrm{a}}$ & $3.4^{\mathrm{a}}$ \\
\hline & 虮子草 Leptochloa panicea & $0.44^{\mathrm{b}}$ & $0.86^{\mathrm{ab}}$ & $2^{\mathrm{a}}$ & $1.7^{\mathrm{ab}}$ & $0.2^{\mathrm{b}}$ \\
\hline & 鳢肠 Eclipta prostrata & $0.8^{\mathrm{abc}}$ & $1.2^{\mathrm{a}}$ & $0.9^{\mathrm{ab}}$ & $0.24^{\mathrm{bc}}$ & $0^{\mathrm{c}}$ \\
\hline & 刺儿菜 Cephalanoplos segetum & $1.1^{\mathrm{a}}$ & $0^{\mathrm{b}}$ & $0^{\mathrm{b}}$ & $0.2^{\mathrm{b}}$ & $0.5^{\mathrm{ab}}$ \\
\hline
\end{tabular}

表中优势度值后的字母表示同一行年度间差异显著 The overall dominancy values followed by different letters in each row are significantly different between the years $(p<0.05)$ 
从表 1 中还可以看出, 旱连作田块在连续 5 年 种植棉花过程中其杂草群落变化较小, 基本稳定, 以 马唐为优势杂草。而在水旱轮作田连续种植 5 年棉 花的过程中，水改旱第一年田块中喜湿性杂草如稗 草、牛繁缕和水花生等杂草的优势度值明显高于旱 连作棉田，而喜旱性杂草如马唐等杂草的优势度值 较旱连作棉田为小, 以后随着棉花种植年数的增加 其与旱连作棉田杂草群落差异逐渐减小, 喜湿性杂 草的优势度值显著降低, 喜旱性杂草的优势度值显 著提高, 而中生性杂草鳢肠优势度值变化不显著。 在水旱轮作田连续种植棉花 4 年期间, 稗草、马唐、 千金子等杂草优势度值与水改旱种植年数之间存在

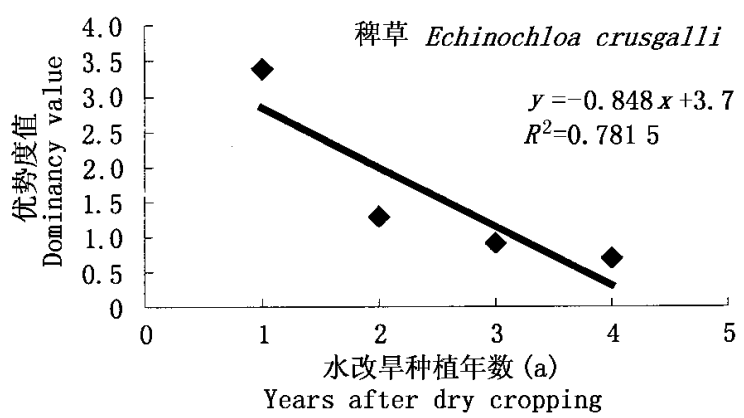

图 1 水改旱 4 年种植年数和稗草优势度值的关系

Fig. 1 Influencing dominance of barnyardgrass by four years continuous dry cropping

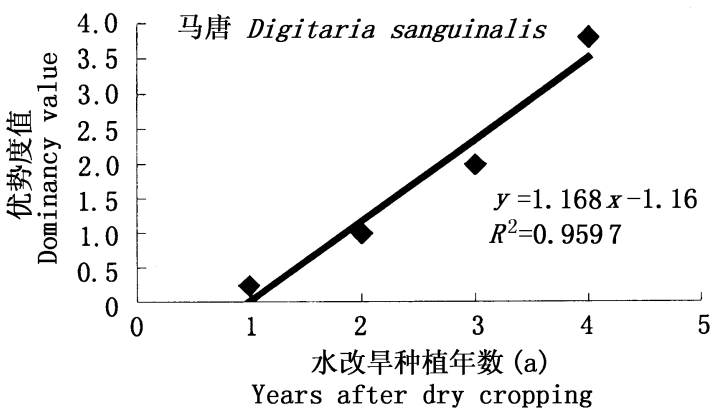

图 2 水改旱 4 年种植年数和马唐优势度值的关系

Fig.2 Influencing dominance of crabdgrass by four years' continuous dry cropping

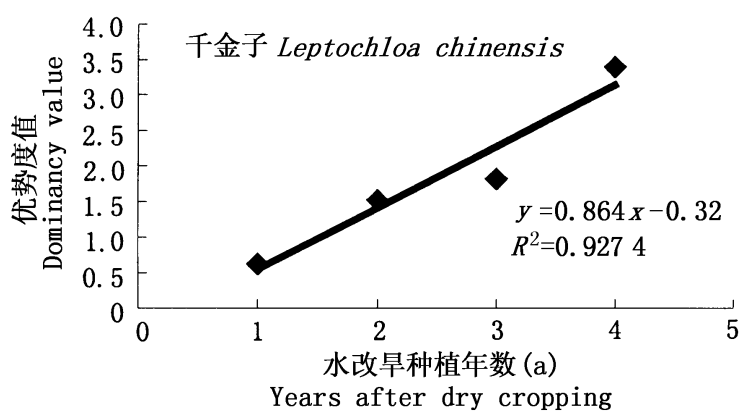

图 3 水改旱 4 年种植年数和千金子优势度值的关系 Fig. 3 Influencing dominance of leptochloa by four years' continuous dry cropping
显著的直线回归关系。喜湿性杂草稗草优势度值随 水改旱种植年数的增加而降低 (图 1);而喜旱性杂 草马唐优势度值则随水改旱种植年数的增加而提高 (图 2、图 3)。通过对连续 5 年种植棉花的水旱轮作 田田间主要杂草优势度值与水改旱种植年数之间的 关系进行直线回归分析 (图 4、图 5、图 6) 后, 发现稗 草、马唐、千金子等杂草优势度值与水改旱种植年数 之间直线回归方程的相关系数相对于水改旱 4 年过 程中的分析结果有较大程度地降低，甚至接近于 0 。 表明水旱轮作田水改旱 4 年内其田间杂草群落变化 显著, 其中, 喜湿性杂草优势度值逐渐减少, 喜旱性 杂草优势度值逐渐提高, 而中生性杂草优势度值变



图 4 水改旱 5 年种植年数和稗草优势度值的关系

Fig.4 Influencing dominance of barnyardgrass by five years' continuous dry cropping

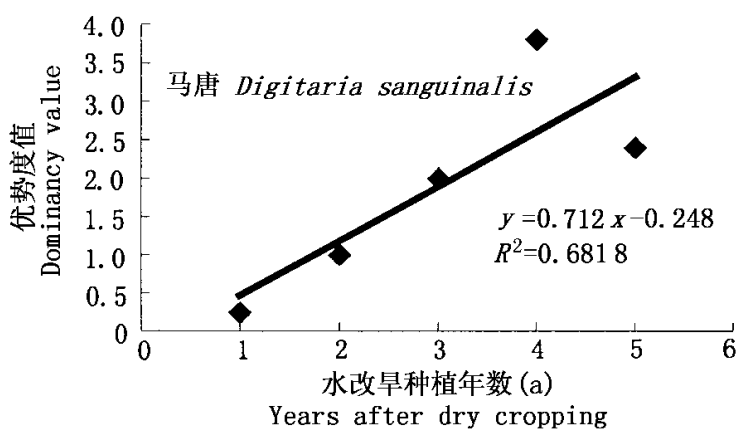

图 5 水改旱 5 年种植年数和马唐优势度值的关系

Fig.5 Influencing dominance of crabdgrass by five years' continuous dry cropping

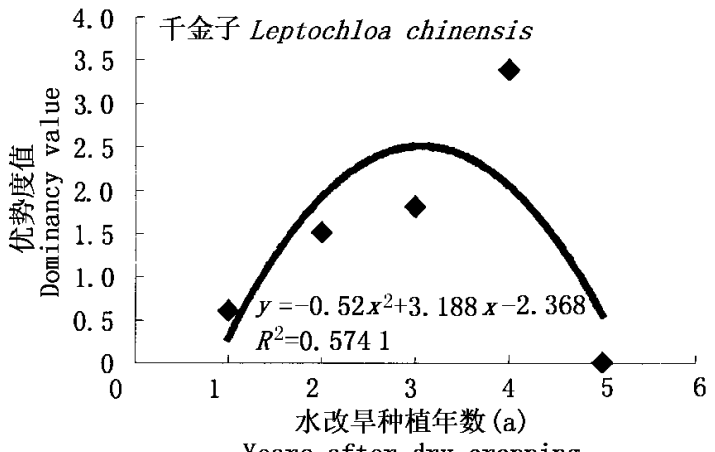

Years after dry cropping

图 6 水改旱 5 年种植年数和千金子优势度值的关系

Fig.6 Influencing dominance of leptochloa by five years' continuous dry cropping 
表 2 种植制度和地理区域两向分组资料的方差分析结果

Table 2 The significance of variance analysis of weed density between different cropping systems and regions

\begin{tabular}{lccc}
\hline & $\begin{array}{c}\text { 杂草株数 }\left(\text { 株 } \mathrm{m}^{-2}\right) \\
\text { Weed density }\end{array}$ & $\begin{array}{c}\text { 差异显著性 } \\
\text { Variance significance level } \\
(p<0.05)\end{array}$ & $\begin{array}{c}\text { 差异显著性 } \\
\text { Variance significance level } \\
(p<0.01)\end{array}$ \\
\hline 旱连作田 Dry cropping field & 95.34 & $\mathrm{a}$ & $\mathrm{A}$ \\
水旱轮作田 Rice and cotton rotation field & 62.56 & $\mathrm{~b}$ & $\mathrm{~B}$ \\
\hline 南通 Nantong & 126.36 & $\mathrm{a}$ & $\mathrm{A}$ \\
宝应 Baoying & 68.59 & $\mathrm{~b}$ & $\mathrm{~B}$ \\
大丰 Dafeng & 41.9 & $\mathrm{c}$ & $\mathrm{B}$ \\
\hline
\end{tabular}

表 3 南通、宝应、大丰三地水旱轮作棉田杂草数量差异显著性分析结果

Table 3 The significance of variance analysis of weed density in the cotton fields with the rice-cotton rotation system in three different places

\begin{tabular}{cccc}
\hline $\begin{array}{c}\text { 地区 } \\
\text { Region }\end{array}$ & $\begin{array}{c}\text { 杂草株数 }\left(\text { 株 } \mathrm{m}^{-2}\right) \\
\text { Weed density }\end{array}$ & $\begin{array}{c}\text { 差异显著性 } \\
\text { Variance significance level } \\
(p<0.05)\end{array}$ & $\begin{array}{c}\text { 差异显著性 } \\
\text { Variance significance level } \\
(p<0.01)\end{array}$ \\
\hline 南通 Nantong & 129.38 & $\mathrm{a}$ & $\mathrm{A}$ \\
宝应 Baoying & 29.4 & $\mathrm{~b}$ & $\mathrm{~B}$ \\
大丰 Dafeng & 28.9 & $\mathrm{~b}$ & $\mathrm{~B}$ \\
\hline
\end{tabular}

表 4 南通、宝应、大丰三地旱连作棉田杂草数量差异显著性分析结果

Table 4 The significance of variance analysis of weed density in the cotton fields with continuous dry cropping rotation in three different places

\begin{tabular}{cccc}
\hline $\begin{array}{c}\text { 地区 } \\
\text { Region }\end{array}$ & $\begin{array}{c}\text { 杂草株数 }\left(\text { 株 } \mathrm{m}^{-2}\right) \\
\text { Weed density }\end{array}$ & $\begin{array}{c}\text { 差异显著性 } \\
\text { Variance significance level } \\
(p<0.05)\end{array}$ & $\begin{array}{c}\text { 差异显著性 } \\
\text { Variance significance level } \\
(p<0.01)\end{array}$ \\
\hline 南通 Nantong & 123.34 & $\mathrm{a}$ & $\mathrm{A}$ \\
宝应 Baoying & 107.78 & $\mathrm{a}$ & $\mathrm{A}$ \\
大丰 Dafeng & 54.9 & $\mathrm{~b}$ & $\mathrm{~A}$ \\
\hline
\end{tabular}

表 5 三地两种种植制度下棉田中同种杂草发生数量差异显著性分析结果

Table 5 The significance of variance analysis of weed density in the cotton fields of three different places under two different cropping systems

\begin{tabular}{|c|c|c|c|c|c|c|}
\hline \multirow{2}{*}{$\begin{array}{c}\text { 杂草种类 } \\
\text { Weed species }\end{array}$} & \multicolumn{2}{|c|}{ 南通 Nantong } & \multicolumn{2}{|c|}{ 宝应 Baoying } & \multicolumn{2}{|c|}{ 大丰 Dafeng } \\
\hline & $\begin{array}{c}\text { 旱连作 } \\
\mathrm{CDC}^{1)}\end{array}$ & $\begin{array}{c}\text { 水旱轮作 } \\
\mathrm{RCR}^{2)}\end{array}$ & $\begin{array}{l}\text { 旱连作 } \\
\mathrm{CDC}^{1)}\end{array}$ & $\begin{array}{c}\text { 水旱轮作 } \\
\mathrm{RCR}^{2)}\end{array}$ & $\begin{array}{l}\text { 旱连作 } \\
\mathrm{CDC}^{1)}\end{array}$ & $\begin{array}{c}\text { 水旱轮作 } \\
\mathrm{RCR}^{2)}\end{array}$ \\
\hline 马唐 Digitaria sanguinalis & $123.3^{\mathrm{a}}$ & $17.7^{\mathrm{c}}$ & $70.7^{\text {abc }}$ & $22^{\text {bc }}$ & $94.7^{\text {ab }}$ & $17.7^{\mathrm{c}}$ \\
\hline 鳢肠 Eclipta prostrata & $5.3^{\mathrm{a}}$ & $4.3^{\mathrm{a}}$ & $3.7^{\mathrm{a}}$ & $8.7^{\mathrm{a}}$ & $3.3^{\mathrm{a}}$ & $11^{\mathrm{a}}$ \\
\hline 铁苋菜 Acalypha australis & $22.7^{\mathrm{a}}$ & $1.3^{\mathrm{b}}$ & $13^{\mathrm{ab}}$ & $3.7^{\mathrm{b}}$ & $11^{\mathrm{ab}}$ & $5^{\mathrm{b}}$ \\
\hline 稗草 Echinochloa crusgalli & $1^{\mathrm{b}}$ & $168^{\mathrm{a}}$ & $2^{\mathrm{b}}$ & $16.3^{\mathrm{b}}$ & $0^{\mathrm{b}}$ & $30.3^{\mathrm{ab}}$ \\
\hline 千金子 Leptochloa chinensis & $6.7^{\mathrm{a}}$ & $10.7^{\mathrm{a}}$ & $0^{\mathrm{a}}$ & $0^{\mathrm{a}}$ & $0^{\mathrm{a}}$ & $3.3^{\mathrm{a}}$ \\
\hline 牛筋草 Eleusine indica & $0^{\mathrm{b}}$ & $2.3^{\mathrm{ab}}$ & $6^{\mathrm{a}}$ & $5.3^{\mathrm{a}}$ & $4^{\mathrm{ab}}$ & $5.2^{\mathrm{a}}$ \\
\hline
\end{tabular}

表中杂草密度后的字母表示同一行三地两种种植制度间差异显著(显著水平取 0.05) The weed density followed by different letters in each row are significantly different between three places and two cropping systems $(p<0.05)$

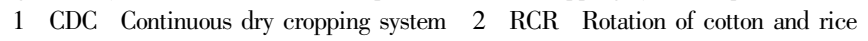

化较小, 水改旱 4 年后其田间杂草群落趋于稳定。

从表 2 列出的南通、宝应、大丰三地及其水旱轮 作、旱连作两种种植制度之间的差异显著性分析结 果可以看出 种植制度是导致江苏省棉田草害发生 差异的第一个因素, 即使在 $p<0.01$ 的情况下水旱 轮作棉田中杂草的发生数量仍然显著低于旱连作棉 田中的杂草发生数量。这说明水旱轮作种植制度能 够显著降低棉田草害的发生程度。另外, 南通、宝 应、大丰三地杂草发生数量之间也存在差异。在显 著水平 $p<0.05$ 的情况下, 南通地区显著高于宝应 地区，宝应地区又显著高于大丰地区; 在显著水平 $p$
$<0.01$ 的情况下, 南通地区显著高于宝应、大丰地 区，而宝应地区和大丰地区之间差异不显著。

从表 3 中列出的南通、宝应、大丰三地水旱轮作 棉田杂草数量差异显著性分析结果可以看出, 南通 地区水旱轮作棉田中杂草发生数量明显高于宝应和 大丰地区; 而从表 4 中又可以看出，南通、宝应、大丰 三地旱连作棉田杂草发生数量差异不明显, 只有在 显著水平取 $p<0.05$ 的情况下, 大丰地区棉田杂草 发生数量才显著低于南通、宝应地区。

从表 5 中可以看出, 在同一种植制度下的不同 地区棉田中同种杂草发生数量差异不显著或较小; 
而在同一地区不同种植制度下的棉田中同种杂草发 生数量差异较为显著。特别是马唐和稗草等优势杂 草的这种趋势明显。这表明, 江苏省棉田中杂草发 生状况受种植制度的影响较大。

\section{3 讨 论}

从以上分析的结果可以看出, 种植制度是影响 江苏省棉田杂草群落的第一个主要因素。

水旱轮作田块在水稻 (Oryza sativa) 种植期间, 通过稻种、猪厩肥和还田稻草将大量的杂草种子带 入田中。在水稻生育期中, 长期淹水条件为田块中 的喜湿性杂草提供了良好的生长发育条件,使其大 量发生。并且在水稻收割前大部分杂草种子已散落 田间,成为来年草种的主要来源 (强胜等, 1997)。 水稻种植期间长期的淹水过程改变了土壤的理化性 质等田间生态条件(朱文达等, 1993) ,使水旱轮作田 块中的水分含量明显高于旱连作田块, 从而导致水 旱轮作田块在种植棉花时田间除了正常生长于旱田 的喜旱性杂草外, 仍有喜湿性杂草的发生。而旱连 作棉田中,田间生态条件基本维持不变, 土壤水分始 终保持较低的状态, 这不适宜喜湿性杂草的生长，从 而导致其棉田中杂草群落结构始终以喜旱性杂草为 主。

旱连作田块一般不会出现长期淹水的现象, 有 利于喜旱性杂草的生长发育, 而使其棉田草害发生 较为严重。而水旱轮作田块由于水稻种植过程中, 长期淹水使原来棉田中生长的喜旱性杂草不能正常 生长发育, 产生繁殖体, 从而降低了翌年种植棉花时 喜旱性杂草种群的发生数量(强胜等, 1999)。而喜 湿性杂草在棉花种植期间由于田间水分含量较低, 其生长发育也受到一定程度抑制。据研究, 喜旱性 杂草狗尾草 (Setaria viridis) 籽实在土壤含水量高于 $40 \%$ 时萌发率降低, 而喜湿性杂草稗草籽实在土壤 含水量 $10 \%$ 以下时很难萌发出苗, 在含水量 $15 \%$ 时 则萌发明显延迟。并且, 水旱轮作棉田的杂草在棉 花播种后的萌发高峰期较旱连作棉田推迟 $10 \mathrm{~d}$ 左 右(吴建荣等,1997)。显然水旱轮作种植制度有抑 制棉田杂草生长发育的作用, 草害发生程度降低。

南通、宝应、大丰 3 个地区总体棉田杂草发生数 量在显著水平取 0.05 的情况下差异显著。可能有 两个方面的因素 :一方面可能是由于上述 3 个地区 的土壤类型、土壤 $\mathrm{pH}$ 值、气温、光照等地理生态因 素的不同间接影响了杂草群落的结构和草害的发 生 ;另一方面, 可能是由于宝应地区采用了地膜覆盖
技术，而大丰地区在棉花移栽前使用了氟乐灵除草 剂，使这两个地区的棉田草害在显著水平取 0.01 的 情况下仍然显著低于南通地区。但三地旱连作棉田 杂草发生数量差异较小, 表明旱连作棉田草害发生 情况受地理区域差异影响较小。虽然, 此前已经研 究报道了除草剂对棉田杂草群落的明显影响, 但是 其研究结果是除草剂使用后的结果, 还是首先引起 群落结构改变后的结果尚不能完全确证 (吴建荣, 2001)。

上述研究结果, 进一步从时间角度研究证明了 江苏省棉田杂草群落发生演替规律及其影响因素主 要受水旱轮作制度的强烈影响, 与此前的全省大范 围的空间调查研究结果形成彼此相互验证的结论 (强胜等，1997；1999；2000)。

\section{参 考 文 献}

Editing Group of Agricultural Geography of Jiangsu(江苏农业地理 编写组). 1979. Agricultural geography of Jiangsu Province. Nanjing: Jiangsu Science and Technology Press. (in Chinese)

Qiang, S. (强胜) \& Y.H. Li (李扬汉). 1990. On the distribution pattern of weed communities of summer crop fields in river valley and hilly lands of Anhui Province. Acta Phytoecologica et Geobotanica Sinica (植物生态学与地植物学学报), 14:212 219. (in Chinese with English abstract)

Qiang, S. (强胜), J.L. Hu(胡金良), J.R. Wu(吴建荣), M. T. He(赫明涛) \& J. Zhang(张军). 1997. The occurrence of weed communities in cotton fields in the regions alongside the Huanghai seacoast of Jiangsu Province. Jiangsu Journal of Agricultural Science(江苏农业学报), 14(2) : 108 111. (in Chinese with English abstract)

Qiang, S. (强胜) \& J. L. Hu (胡金良). 1999. Quantitative analysis of the weed communities of the cotton fields in the cottongrowing regions of Jiangsu Province. Acta Ecologica Sinica(生态 学报), 19:810 816. (in Chinese with English abstract)

Qiang, S. (强胜), S.H. Wei(魏守辉) \& J. L. Hu(胡金良). 2000. On weed flora and infestation in cotton fields in Jiangsu Province. Journal of Nanjing Agricultural University(南京农业 大学学报), 23(2): 18 22. (in Chinese with English abstract) Wu, J.R. (吴建荣), S.M. Zhang(张绍明), Y.S. Wang (王永 山), G.Y. Shao(邵耕耘), M.G. Li(李明光) \& W.L. Tang (唐维林). 1997. Weed occurrence and affecting factors in the transplant cotton fields. Weed Science (杂草科学), (2) : 15 17. (in Chinese)

Wu, J.R. (吴建荣), R.L.Ji(吉荣龙), B.B. Cui(崔必波) \& C.H. Li (李春宏). 2001. Effects of herbicides on weed community structure in cotton field. Jiangsu Journal of Agriculture Science(江苏农业学报)，17(1):28～33. (in Chinese with English abstract)

Zanin, G., S. Otto, L. Riello \& M. Borin. 1997. Ecological interpretation of weed flora dynamics under different tillage systems. Agriculture, Ecosystems and Environment, 66:177 188 .

Zhu, W.D. (朱文达) \& X.H. Ma(马小华). 1993. Influence of rice-cotton rotation on weeds and weed communities. Journal of Weed Science(杂草学报),7(3):5 10. (in Chinese with English abstract) 